\title{
Progress in the Development of Mo/Au Transition-Edge Sensors for X-ray Spectroscopy
}

\author{
Caroline K. Stahle $^{\dagger a}$, Regis P. Brekosky ${ }^{\mathrm{a}, \mathrm{b}}$, Enectali Figueroa-Feliciano ${ }^{\mathrm{a}, \mathrm{c}}$, Fred M. Finkbeiner ${ }^{\mathrm{a}, \mathrm{d}}$, \\ John D. Gygax ${ }^{\mathrm{a}, \mathrm{b}}$, Mary J. Li ${ }^{\mathrm{a}, \mathrm{e}}$, Mark A. Lindeman ${ }^{\mathrm{a}, \mathrm{f}}$, F. Scott Porter ${ }^{\mathrm{a}}$, Nilesh Tralshawala, \\ ${ }^{a}$ NASA /Goddard Space Flight Center, Greenbelt, MD 20771 \\ ${ }^{\mathrm{b}}$ Swales Corporation, Beltsville, MD \\ ${ }^{\mathrm{c}}$ Physics Department, Stanford University \\ ${ }^{\mathrm{d}}$ Department of Astronomy, University of Maryland \\ ${ }^{\mathrm{e}}$ Raytheon ITSS, Lanham, MD \\ fNAS/NRC Resident Research Associate
}

\begin{abstract}
X-ray microcalorimeters using transition-edge sensors (TES) show great promise for use in astronomical x-ray spectroscopy. We have obtained very high energy resolution $(2.8 \mathrm{eV}$ at $1.5 \mathrm{keV}$ and $3.7 \mathrm{eV}$ at $3.3 \mathrm{keV})$ in a large, isolated TES pixel using a $\mathrm{Mo} / \mathrm{Au}$ proximity-effect bilayer on a silicon nitride membrane. We will discuss the performance and our characterization of that device. In order to be truly suitable for use behind an x-ray telescope, however, such devices need to be arrayed with a pixel size and focal-plane coverage commensurate with the telescope focal length and spatial resolution. Since this requires fitting the TES and its thermal link, a critical component of each calorimeter pixel, into a far more compact geometry than has previously been investigated, we must study the fundamental scaling laws in pixel optimization. We have designed a photolithography mask that will allow us to probe the range in thermal conductance that can be obtained by perforating the nitride membrane in a narrow perimeter around the sensor. This mask will also show the effects of reducing the TES area. Though we have not yet tested devices of the compact designs, we will present our progress in several of the key processing steps and discuss the parameter space of our intended investigations.
\end{abstract}

Keywords: microcalorimeter, x-ray spectroscopy, imaging spectrometer

\section{INTRODUCTION}

There is considerable interest in optimizing superconducting transition-edge sensor (TES) microcalorimeters for astrophysical x-ray spectroscopy. Since most superconductors undergo the transition to zero resistance at temperatures too high to be of use in a microcalorimeter, it is common to use a bilayer of normal and superconducting films. Through the proximity effect, the composite film behaves as a single superconductor with a lower transition temperature than the superconducting layer would have had alone. The choice of metals is guided by the need to survive the temperature changes and chemical exposures to which the devices will be exposed en route to becoming a focal plane array, as well as optimizing the superconducting properties. At Goddard, we have been developing bilayers composed of molybdenum and gold. ${ }^{1}$ No intermetallic compound formation at the $\mathrm{Mo} / \mathrm{Au}$ interface is expected for temperatures below $800{ }^{\circ} \mathrm{C}$ and negligible interdiffusion is anticipated through the interface for temperatures less than $300{ }^{\circ} \mathrm{C}^{2,3}$ Because the constituents are immiscible and non-reactive, Mo/Au bilayers promise long-term stability.

In order to evaluate $\mathrm{Mo} / \mathrm{Au}$ bilayers as thermometers in microcalorimeters, we have incorporated them into simple calorimeter structures that use silicon nitride membranes for the thermal isolation to the heat sink. The area of one of these membranes is larger than the area of the TES thermometer itself. Even before we obtained high spectral resolution with one of these test structures, we began planning for arrays of closely packed TES x-ray calorimeters. Given a pixel area specified by the x-ray optics, how do we best allocate the available space between the TES, the thermal link, and area for electrical

\footnotetext{
${ }^{\dagger}$ corresponding author: Caroline.K.Stahle@gsfc.nasa.gov
} 
contacts? What is the smallest area that the link can occupy and still provide adequate isolation? The first steps toward TES arrays lie in the answers to these questions.

\section{PERFORMANCE AND CHARACTERIZATION OF AN ISOLATED, SINGLE PIXEL}

\subsection{Fabrication, test apparatus, and specific detector configuration}

We start with silicon wafers that have $0.5 \mu \mathrm{m}$ of low stress silicon nitride deposited on the surface and etch through the Si to define nitride membranes. (The low stress composition is non-stoichiometric; we will abbreviate silicon nitride as Si-N in the rest of this paper.) Presently we are using electron-beam evaporation in an ultra-high vacuum deposition system to deposit the Mo and $\mathrm{Au}$ layers. We use sputtered $\mathrm{Nb}$ for the electrical contacts to the Mo/Au TES. Both chemical etching and liftoff are used at stages in the device patterning. The Mo is further etched so that the edge of the Au layer overhangs the edge of the Mo. We had found that this treatment led to reproducibly sharp transitions. Details pertaining to the fabrication can be found in Ref. 4. In the near future, we will deposit a bismuth x-ray absorber directly on the suspended TES bilayer. We are in the process of characterizing the $\mathrm{Bi} / \mathrm{Au}$ interface that results from this step and thus have not yet incorporated it into devices.

We use a dilution refrigerator to provide a regulated heat sink as low as $50 \mathrm{mK}$ for our TES calorimeters. The dilution refrigerator resides in an electromagnetic shield room. While we have not yet seen any change in detector response for operating with the screen room door open, this layout does serve to situate the noisy electronics (e.g. computer monitors) at a distance. The dewar sits in a mu-metal bucket for magnetic shielding. The detectors are further shielded from magnetic fields by an aluminum can. Since the dewar has no window for x-rays, radioactive sources are mounted inside the aluminum can. We use ${ }^{41} \mathrm{Ca}$ for lines at 3.3 and $3.6 \mathrm{keV}$. An ${ }^{55} \mathrm{Fe}$ source configured to fluoresce an $\mathrm{Al}$ target provides 1.5, 5.9, and $6.5 \mathrm{keV}$ lines.

The apparatus is presently wired for four independent TES channels. Series arrays of DC SQUIDs are mounted off the base plate just below the continuous heat exchanger of the dilution refrigerator. Twisted pairs of $\mathrm{Cu} / \mathrm{Ni}$ clad $\mathrm{Nb} / \mathrm{Ti}$ wire connect the TES bias circuit at the mixing chamber with the SQUID input coil. Parasitic resistance in the bias circuit is generally only a few $\mu \Omega$. There is a $2 \mathrm{~m} \Omega$ bias resistor for each TES and input coil pair. Current is supplied to the parallel combination of this bias resistor with the TES and input coil through an external resistor by a tunable, quiet voltage supply. The SQUID bias and feedback electronics are mounted on the dewar support structure.

Although our single pixel test devices cover a range of TES and membrane areas, we will be presenting the results from one particular device. It consists of a $0.5 \mathrm{~mm} \times 0.5 \mathrm{~mm}$ TES $(50 \mathrm{~nm} \mathrm{Mo}, 270 \mathrm{~nm} \mathrm{Au})$ with $0.5 \mathrm{~mm}$ wide $\mathrm{Nb}$ leads on a $5 \mathrm{~mm} \times$ $5 \mathrm{~mm} \times 0.5 \mu \mathrm{m}$ low stress Si-N membrane. The normal state resistance $R_{n}=10.2 \mathrm{~m} \Omega$, and the superconducting transition temperature $T_{c}=106 \mathrm{mK}$ for low current. As we already indicated, it had no additional x-ray absorber layer. The electronics channel for this device used a 100-SQUID series array provided by N. Bergren of NIST - Boulder and M. E. Huber of the University of Colorado at Denver. The ratio of the input coil coupling to the feedback coil coupling was 3.2.

The thermal conductance of the membrane at the operating point was $\sim 3 \times 10^{-10} \mathrm{~W} / \mathrm{K}$. Assuming that the thermal conductance of the membrane can be described by the equation $G(T)=n K T^{n-1}$, we can use the dependence on the heat sink temperature of the power required to bias to a particular point in the transition to determine both $n$ and $T_{c}$. It is hard to fit the entire temperature range from $50 \mathrm{mK}$ to $T_{c}$, possibly due to shifting of $T_{c}$ with current. The best fit from $50 \mathrm{mK}$ to $86 \mathrm{mK}$ gives $n=2.6$, but also gives $103 \mathrm{mK}$ for $T_{c}$.

The parameter $\alpha=d \log (R) / d \log (T)$ defines the sensitivity of the TES thermometer. Four-wire measurements on Mo/Au test coupons from the same deposition, on solid substrates rather than membranes to minimize runaway self-heating, determined mid-transition values for $\alpha$ of $\sim 400$. A series of curves plotting TES current versus applied bias current at different heat sink temperatures was obtained for the device studied. Starting from the normal state and high biases and going to lower bias, the relationship between the two currents proceeds as expected for a resistance of $10.2 \mathrm{~m} \Omega$ until the temperature drops to $T_{c}$. At that point the resistance begins to drop but the current through the TES increases. The power to maintain the TES in its transition varies little across a narrow transition, thus a reduction in resistance requires an increase in current. Negative electrothermal feedback, the effect that an increase in temperature results in a decrease in Joule power, makes it possible for the TES to self-regulate at a particular resistance within the transition. As the bias is reduced further, however, and the TES 


\begin{tabular}{|c|c|c|c|c|c|}
\hline $\begin{array}{c}\text { fraction of } \\
\text { normal resistance }\end{array}$ & $\begin{array}{c}\text { approximate extent } \\
\text { of linear range }\end{array}$ & $\begin{array}{c}\text { baseline } \\
\text { FWHM in eV }\end{array}$ & $\begin{array}{c}1.5 \mathbf{~ k e V} \\
\text { FWHM in eV }\end{array}$ & $\begin{array}{c}\mathbf{3 . 3} \mathbf{~ k e V} \\
\text { FWHM in eV }\end{array}$ & $\begin{array}{c}\mathbf{5 . 9} \mathbf{~ k e V} \\
\text { FWHM in eV }\end{array}$ \\
\hline 0.8 & $2.5 \mathrm{keV}$ & 2.6 & 2.8 & undefined & undefined \\
\hline 0.66 & $4.0 \mathrm{keV}$ & 3.5 & 3.4 & 4.2 & undefined \\
\hline 0.6 & $5.0 \mathrm{keV}$ & 3.7 & 3.7 & 3.7 & undefined \\
\hline 0.55 & $6.0 \mathrm{keV}$ & 4.5 & 4.7 & 4.4 & poorly defined \\
\hline 0.47 & $7.5 \mathrm{keV}$ & 5.0 & 5.1 & 5.0 & 6.1 \\
\hline
\end{tabular}

Table 1. Resolution obtained at different energies for different bias points within the superconducting transition. The heat sink temperature was $50 \mathrm{mK}$.

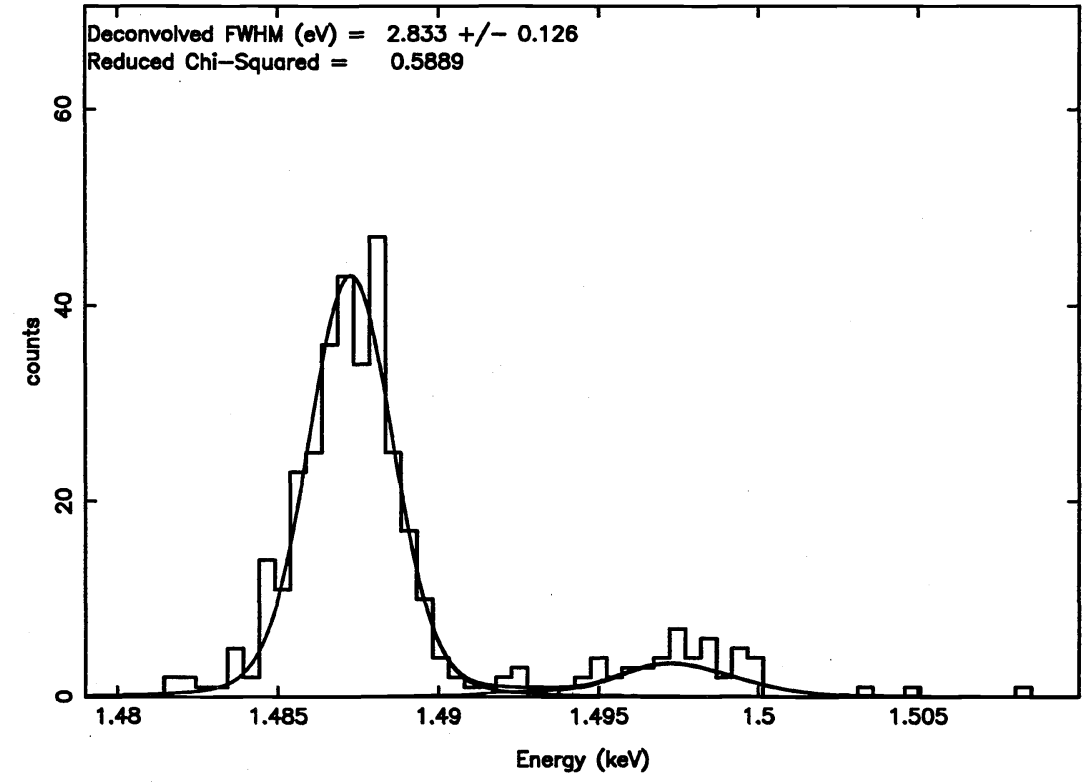

Figure 1-2.8 eV resolution obtained for a bias near the top of the transition

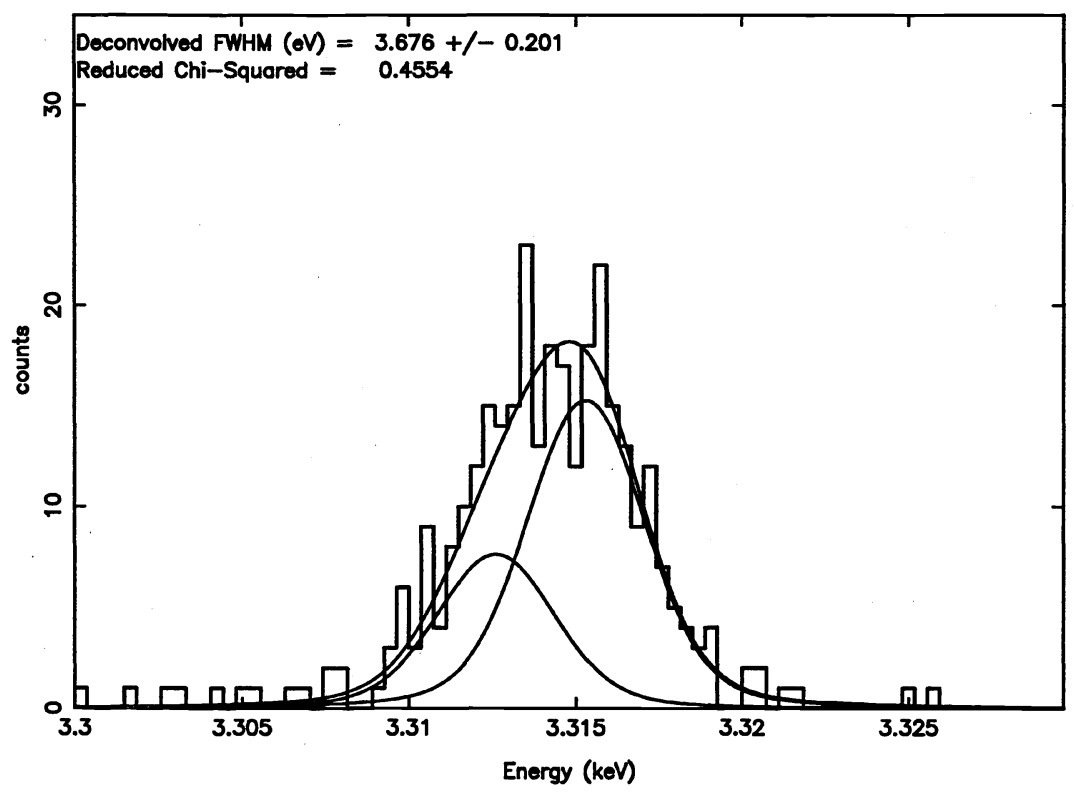

Figure 2-3.7 eV resolution obtained for a mid-transition bias resistance drops below that of the bias resistor, the feedback switches from negative to positive, and the TES snaps to the superconducting state. The small amount that the power does change while traversing the accessible part of the transition can be used, with the thermal conductance function, to assign a temperature to each resistance. The curve taken at a heat sink temperature of $50 \mathrm{mK}$, the temperature at which we acquired most of our spectra, was applied in this way to estimate $\alpha$ under operating conditions, and this also yielded $\alpha \sim 400$ for reasonable values of $n$.

\subsection{Resolution}

We experimented with the configuration of the electronics after the SQUID controller, but the following description is typical of most of the resolution measurements. Spectra were acquired by running the feedback signal from the SQUID controller into a pre-amplifier with gain of 100 and then into a 12-bit digital oscilloscope. We typically used a $10 \mu \mathrm{s}$ sample interval, and a two-pole roll-off at $30 \mathrm{kHz}$ was applied at the preamplifier as an anti-aliasing filter. Triggered records of 4096 or 8912 samples, including $\sim 20 \%$ pretrigger, were saved to a computer file. Every fifth record was a randomly triggered sweep meant to capture the noise so that an optimal digital filter could be applied to the data. The excessive length of the records was chosen to aid in resolving pick-up at $60 \mathrm{~Hz}$ and its harmonics. Though we minimized its contribution with careful grounding, we wanted to be able to measure whatever still remained in as little of the signal bandwidth as 
feasible. The pulse itself decayed to baseline well before the end of the record. The exponential fall time of a pulse for a bias near the middle of the transition was $0.31 \mathrm{~ms}$.

We accumulated pulses for different bias points in the transition. At higher bias, the dynamic range of the TES is restricted because the starting resistance is close to the normal resistance. At lower bias, the dynamic range is larger, but the correspondingly higher current could broaden the transition there and introduce excess noise. Thus, we chose the bias points to span the range from having only the $1.5 \mathrm{keV}$ line in the linear range to having linear response all the way through $6.4 \mathrm{keV}$. For each data set we derived and applied an optimal filter. In some cases, a small amount of gain drift in the resulting filtered event list was also corrected. Table 1 summarizes the full-width-at-half-maximum (FWHM) line widths obtained from these measurements after deconvolving the natural line widths from the instrument response. The $1 \sigma$ error on the line width determinations was typically $0.2 \mathrm{eV}$. The "baseline" FWHM refers to a Gaussian fit to the distribution of values obtained from applying the optimal filter to the noise sweeps. That the line widths are largely consistent with the baseline noise indicates that thermalization noise and long-term gain variations are negligible. We have identified each bias point in the table by the resistance, expressed as a fraction of the normal resistance, and by an estimate of the extent of the linear region. We sacrifice resolution at the lower energies in order to get the higher energy lines on scale. Figures 1 and 2 illustrate the $2.8 \mathrm{eV}$ resolution fit to $\mathrm{Al} \mathrm{K} \alpha$ fluorescence and the $3.7 \mathrm{eV}$ resolution fit to the $\mathrm{K} \mathrm{K} \alpha$ fluorescence. Five Lorentzian lines were fit to the $1.5 \mathrm{keV} \mathrm{Al}$ fluorescence, using the line widths and energies appropriate for the case of elemental $\mathrm{Al}$, as discussed in references 5 and 6 . The position and widths of the $\mathrm{K} \alpha_{1}$ and $\mathrm{K} \alpha_{2}$ Lorentzians for $\mathrm{K}\left(3.3 \mathrm{keV},{ }^{41} \mathrm{Ca}\right)$ were taken from reference 7 and for $\mathrm{Mn}\left(5.9 \mathrm{keV},{ }^{55} \mathrm{Fe}\right)$ from reference 8 .

What energy resolution should we have expected? We need to know $C / \alpha$ (where $C$ is the heat capacity) and $n$ to calculate the theoretical resolution, but the resolution is only weakly dependent on $n .^{9}$ We can calculate $C / \alpha$ from the response to a photon of known energy. For the $1.5 \mathrm{keV}$ photons, the pulse height was nearly independent of bias over the range of bias points shown in Table 1. This pulse height gives us $C / \alpha=1.7 \times 10^{-14} \mathrm{~J} / \mathrm{K}$ for the $6.1 \mathrm{~m} \Omega$ bias, and this ratio differs only slightly at the other bias points. (For the expected $C$ for this system, $\alpha \sim 100$ ). An ideal calorimeter with the same $C / \alpha$ would get $\sim 1.6 \mathrm{eV}$ resolution. We're not doing too badly compared with that, but what is the source of the discrepancy? And, given the presumed sharpness of our transition, why isn't $\alpha \sim 400$ ?

\subsection{Excess noise}

The resolution degrades as the TES is biased lower in the transition because there is a non-ideal noise source that increases with lower bias. Recall that an ideal calorimeter has only two sources of intrinsic noise: Johnson noise and thermodynamic fluctuation noise called "phonon noise". 10,11 The magnitude of the phonon noise has the same frequency distribution as the signal. If there were no other noise, the signal-to-noise ratio would be frequency-independent, and since the frequency components of the signal are correlated but those of the noise are independent and add in quadrature, the measurement error could be made arbitrarily small by employing a sufficiently large bandwidth. In the case of an ideal resistance-based calorimeter, the Johnson noise of the sensor limits the bandwidth over which the signal-tonoise ratio is high. Thermalization and diffusion times also suppress the signal relative to the phonon noise at high frequencies. In calorimeters with large $\alpha$, both the signal and the phonon noise magnitude are elevated with respect to the Johnson noise. Thus, at low frequencies, we expect to measure a noise level consistent with the phonon noise current. That noise will fall off above a corner frequency $1 / \tau_{\text {eff }}$, where $\tau_{\text {eff }}$ is the time constant of the thermal recovery, until it hits the Johnson noise. The crossover point of the phonon noise and the Johnson noise effectively defines the measurement bandwidth when an optimal filter is applied.

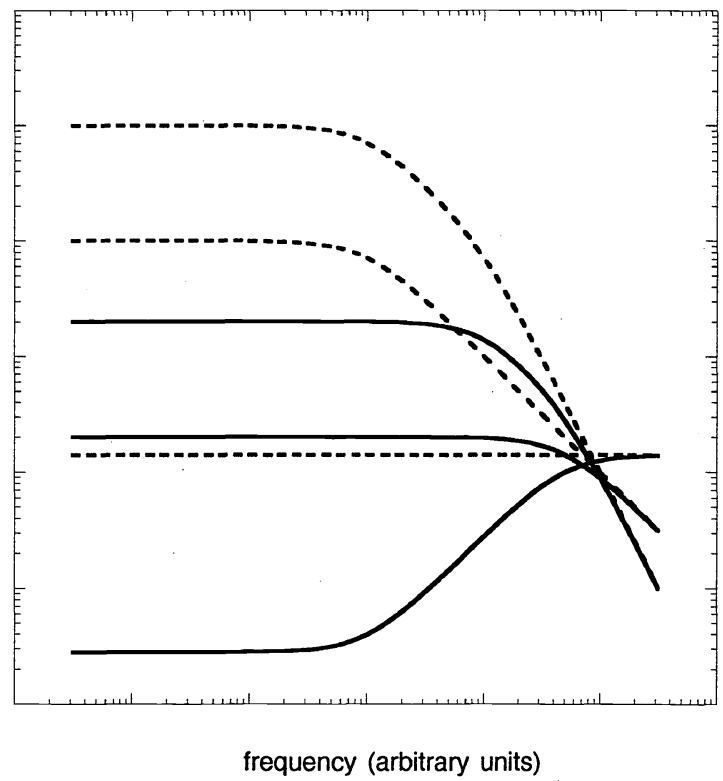

Figure 3 - Signal, phonon noise, and Johnson noise without electrothermal feedback (shown in dashed lines) and with a large electrothermal feedback gain (shown in solid lines). 


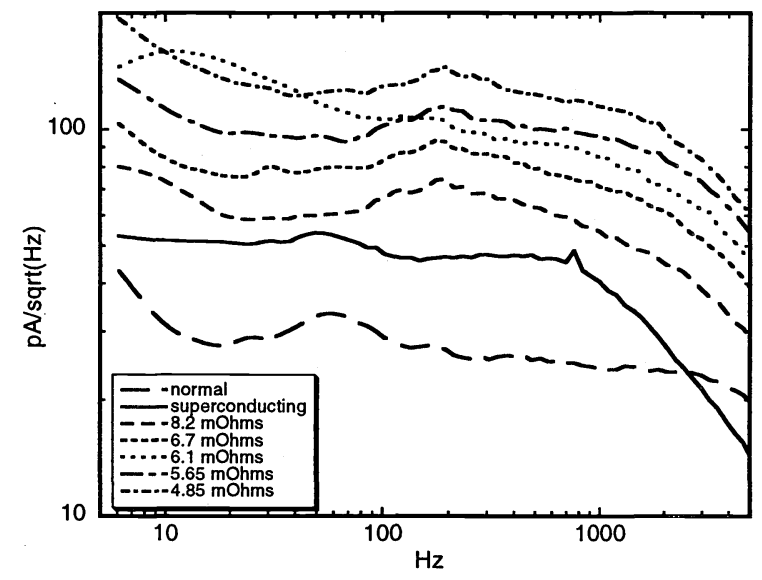

Figure 4 - Smoothed noise spectra for different bias points within the transistion for a $50 \mathrm{mK}$ heat sink.

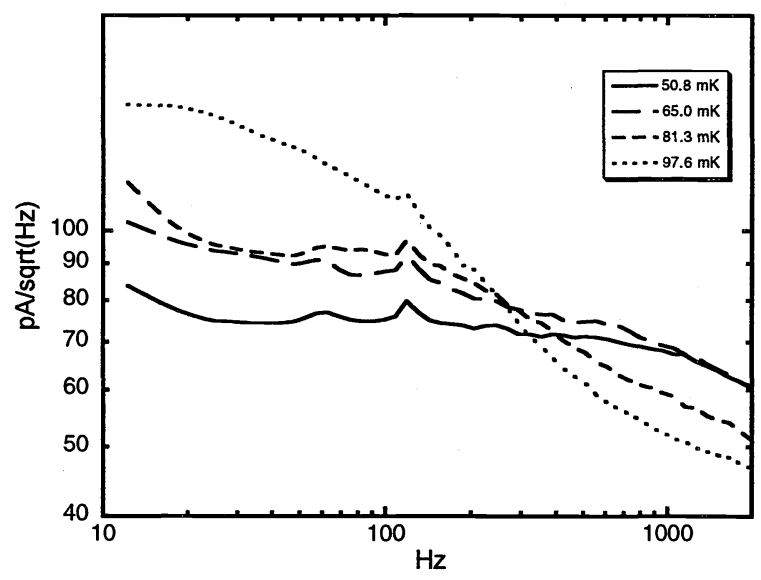

Figure 5 - Smoothed noise spectra for different heat sink temperatures but with the TES biased at the same resistance. Note how the phonon noise increases and the mid-frequency excess noise decreases with increasing temperature. oise was apparently suppressed below $1 / \tau_{e f f}$, as would have been expected for any noise source doing work on the TES. For a heat sink temperature of $50 \mathrm{mK}$, the excess noise increased with the phonon noise, leaving the noise spectrum surprisingly white out to the $L / R$ roll-off, though well above the Johnson noise level. This result is illustrated in Figure 4 . For display purposes only, in order to make the trend clear in a black-and-white plot, we excluded frequency bins, e.g. $60 \mathrm{~Hz}$, with line interference and then applied a smoothing function to the remaining points. This plot also shows the noise measured when the device was normal and superconducting. The Johnson noise for all the bias points would lie between those two curves. Clearly the Johnson noise while in the transition is dominated by excess noise. Thus this excess, which at first seems "out of band" and harmless, is actually within the optimal filter band of the ideal detector. Because $1 / \tau_{\text {eff }}$ decreases as we approach the resistance of the shunt, this noise restricts the high-signal-to-noise bandwidth to an ever decreasing band as the TES is biased further down in the transition, hence the degradation in resolution.
When combined with a constant voltage bias and a heat sink temperature much colder than the detector operating temperature, a large $\alpha$ results in large negative electrothermal feedback gain. Large feedback gain makes the temperature recover faster, after an excursion from quiescence, and it is useful to speed the recovery up to the point that the corner frequency of the thermal response matches the useful bandwidth of the Electrothermal feedback does not change entrement bandwidth, so long as amplifier signal and the frequencies to achieve faster recovery times. This effect is illustrated in Figure 3. For the case of voltage biasing, heat sink temperature much less than $T_{c}$, and $\alpha / n>>1$, the low frequency phonon noise level in amps/sqrt(Hz) asymptotes to a level only $\operatorname{sqrt}(n / 2)$ larger than the unsuppressed Johnson noise level. In this case, the noise is nearly white up to the $L / R$ roll-off due to the SQUID input inductance and parasitic defined by $1 / \tau_{\text {eff }}$.

This is not the situation for our set-up. Because the shunt resistor for the read-out channel of this detector was $2.3 \mathrm{~m} \Omega$, compared with the $10.2 \mathrm{~m} \Omega$ normal state resistance, the TES was not perfectly voltage biased and became less so as the resistance of the bias point approached the value of the shunt resistor. In this situation we expect to see the phonon noise level increase and its corner frequency decrease as the bias point descends further into the transition. Indeed, we did see the phonon noise level increase as the bias was decreased, in the manner expected, but we were never able to see the Johnson noise level. Additional noise far in excess of the Johnson noise was seen in the mid-frequency range between $1 / \tau_{\text {eff }}$ and the $L / R$ roll-off. Because the low frequency noise level was very close to that expected for the phonon noise alone, this excess
for any noise source doing work on the TES. For 


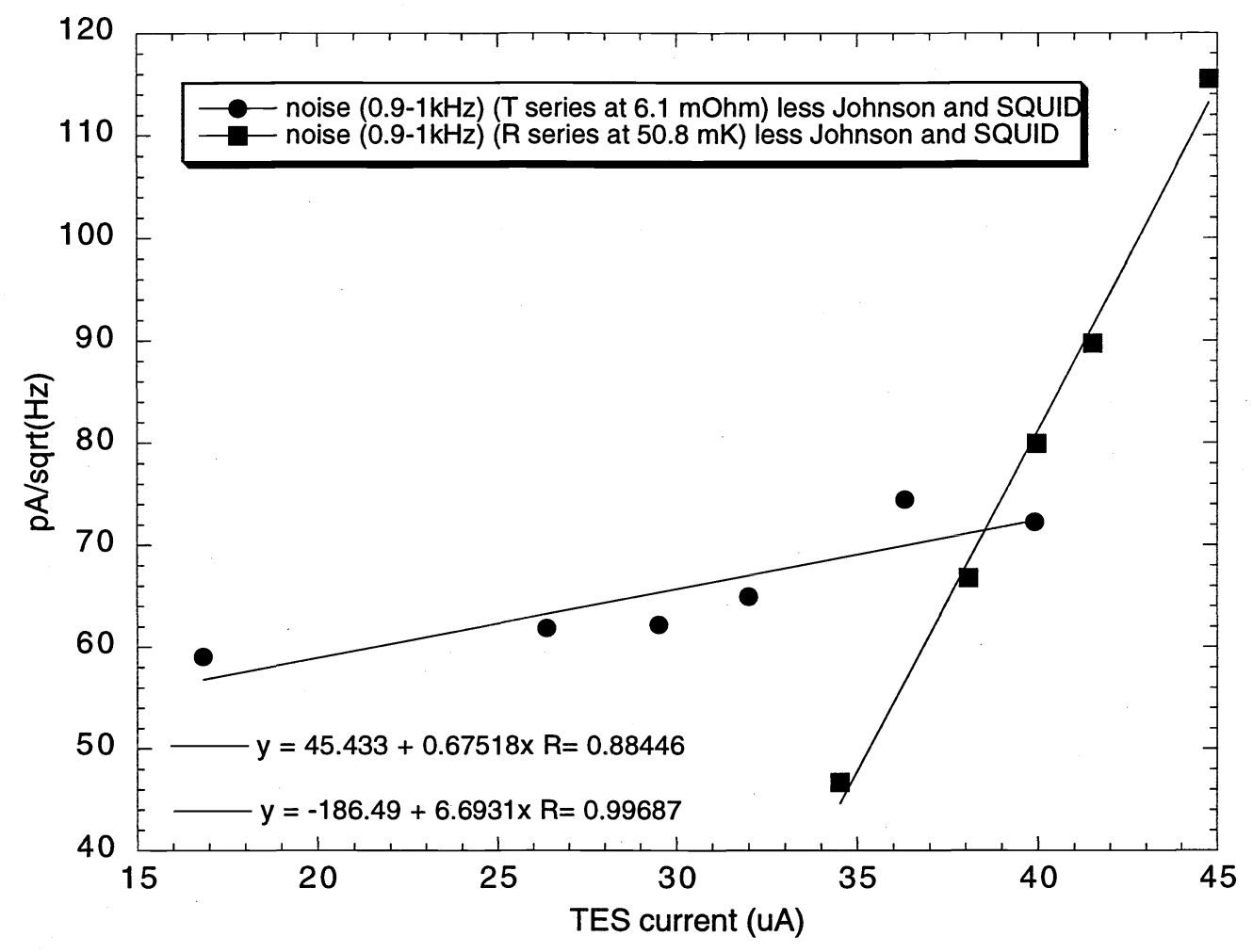

Figure 6 - Plot of excess noise versus current for two different ways of varying the current. The squares are for constant heat sink temperature and different resistances within the transition. The circles are for constant resistance within the transition, and varied heat sink temperature.

For a particular heat sink temperature, the current through the TES increases as it is biased at lower resistances. We have also mapped the excess noise as a function of current by varying the heat sink temperature but adjusting the bias current to keep the TES at a particular resistance in the transition, in this case $6.1 \mathrm{~m} \Omega$. The result of that investigation is shown in Figure 5. Again, for display purposes, we have applied a smoothing function to the curves. As the bath temperature is lowered, the phonon noise increases and the excess noise decreases. Finally, we actually observe a $\tau_{\text {eff }}$ fall-off. The phonon noise increases with bath temperature for two reasons. First of all, as the heat sink goes from $0 \mathrm{~K}$ to $T_{c}$, the phonon noise power itself increases by sqrt(2). In addition, the feedback gain is diminished as the temperature difference is reduced.

We have combined Figure 4 and Figure 5 (and two temperatures not included in Figure 5 to simplify the plot) into Figure 6 , which plots the excess noise (averaged over 900 to $1000 \mathrm{~Hz}$ and with the Johnson noise and previously measured SQUID noise subtracted off) versus the current flowing in the TES. The frequency band was chosen to avoid interference lines and both the $\tau_{e f f}$ and $L / R$ roll-offs. The data taken at a $50 \mathrm{mK}$ heat sink temperature are plotted as squares, and those taken at the $6.1 \mathrm{~m} \Omega$ point for different bath temperatures are shown as circles. Clearly the excess noise is not determined by current alone; it appears more dependent on the particular point in the transition. Our hopes that we could improve the resolution in the high-dynamic-range cases by increasing the bath temperature were shown to be unfounded by this result. Further study is required to address the physical origin of the excess noise. 


\section{REQUIREMENTS OF A CLOSE-PACKED ARRAY}

In the present baseline design of the Constellation- $X \mathrm{x}$-ray imaging spectroscopy mission, there is a calorimeter array with at least $30 \times 30$ elements. The width of each square pixel is $0.25 \mathrm{~mm}$. TES calorimeters are the leading technology for this array, and our plans to make TES arrays have been guided by the Constellation- $X$ requirements.

\subsection{Cantilevered absorbers}

Within each $0.25 \mathrm{~mm}$ unit cell, we must accommodate a TES thermometer, a thermal link, and a channel for bringing out the electrical contacts from that pixel and others. While the apportionment between these items is the topic of the next section, in this section we will address the thermalizing $\mathrm{x}$-ray absorber, which lies above these other components. The absorber clearly is attached only to the TES, otherwise it would provide a thermal short across the weak link. But the absorber also defines the active area of a pixel. In order to minimize loss of photons in the gaps between pixels, the absorber must fill as much of the unit cell as feasible. This requires an absorber that is in contact with the thermometer, but then extends cantilevered over the remaining area of the pixel.

We are refining a process for producing $\mathrm{Bi}$ absorbers that make contact to a substrate through a central stem, but extend cantilevered above the substrate in an overhanging ledge supported by the stem (like the cap of a mushroom). We had promising early success ${ }^{12}$ with the use of patterned photoresist absorber molds and a lift-off process for making closely packed arrays of $\sim 10 \mu \mathrm{m} \mathrm{Bi}$ and $\mathrm{Bi} / \mathrm{Cu}$ "mushrooms". The thickness in the stem and the overhanging ledge is the same, but the surface of the ledge is higher because it is elevated above the substrate. We have produced intact $32 \times 32$ arrays of such absorbers, in a variety of stem sizes and ledge sizes, on solid Si substrates and on Si-N membranes. We have also demonstrated a process that allows the photoresist mold to be patterned on a membrane that has been perforated. The back of the membrane is coated with Al prior to the reactive ion etching of the pattern of slits in the membrane. The Al backing bridges those slits during the patterning of the $\mathrm{Bi}$ lift-off mold and is removed before actual liftoff. This procedure has been validated on $\mathrm{Si}$ membrane structures and we plan to apply it to slit Si-N membranes soon. Figure 7, from Reference 12 , illustrates the concept.

a)
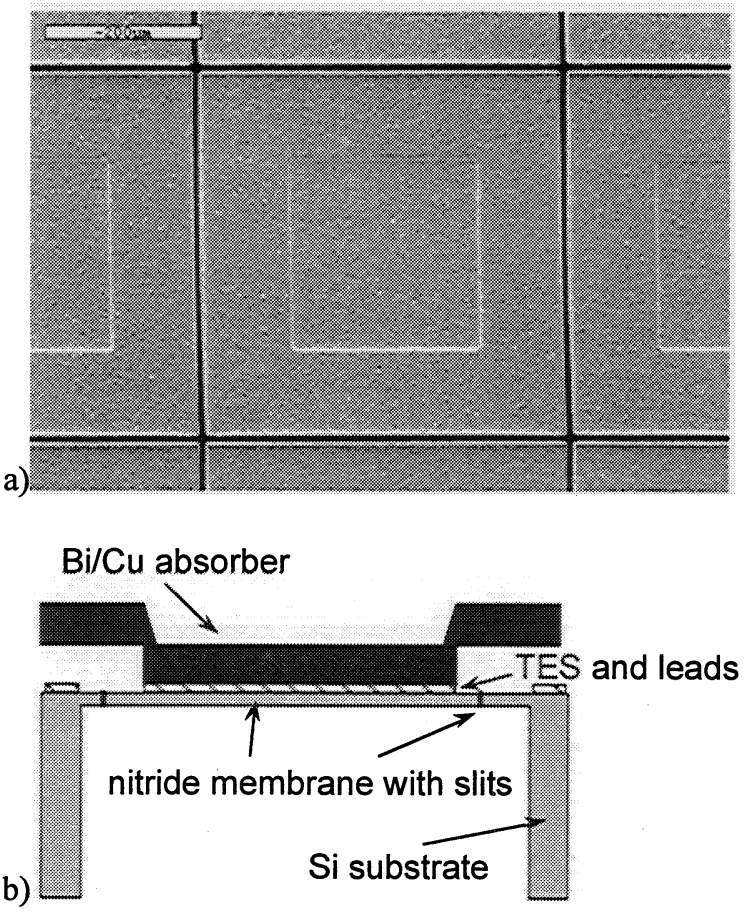

Figure 7 - a) Top view of segment of array of $\mathrm{Bi} / \mathrm{Cu}$ absorbers. The area occupied by the "stem" is $0.2 \mathrm{~mm}$ wide and the extent of the overhang is $0.1 \mathrm{~mm}$.

b) Schematic showing cross section of proposed TES calorimeter pixel using such an absorber

\subsection{Compact pixels}

The Constellation- $X$ design requires fitting the TES and its thermal link into a far more compact geometry than has previously been investigated, thus we must study the fundamental scaling laws that govern the TES and the weak thermal link. If we were to decrease the area of the TES thermometer we've been discussing, but leave the other parameters the same, we would increase the current density for a given bias point, and we would decrease the heat capacity. The width of a superconducting transition is typically narrower at lower current densities than at higher densities. If this broadening scaled inversely with volume such that $C / \alpha$ remained constant, then we could tolerate this reduction in $\alpha$. If the excess noise depends on current density, however, then limits on the noise will place limits on the current density and determine the maximum value of the thermal conductance of the weak link, since that parameter determines the bias power required to operate at the TES transition temperature. The more current a TES can handle before the onset on non-ideal behavior, the faster the device can be designed to operate. ${ }^{13}$ Thus, the size constraint tends to work against the 1000 -counts-per-pixel 
requirement of Constellation- $X$. On the same wafer that yielded the single-pixel results of this paper we have test devices with smaller TES thermometers, and we plan to investigate the behavior of those devices. Our new photolithography mask for array studies also includes two different TES sizes, one $0.15 \mathrm{~mm}$ that fits within the $0.25 \mathrm{~mm}$ unit cell, and one $0.3 \mathrm{~mm}$ designed for a $0.4 \mathrm{~mm}$ pixel.

For a bilayer, there is a limited amount of room for expansion in the vertical direction within the bounds of the proximity effect. Applying additional layers of comparable thickness removes that limit, but leaves us with a TES with a very small normal resistance. The inductance in the TES bias circuit would need to be dropped by the same factor as the resistance in order to avoid electrothermal instability. This constraint requires using a SQUID with smaller input coupling (the larger current signal and Johnson noise due to the lower resistance compensate for the corresponding increase in input-referred noise) and tight tolerances on parasitic inductance in the wiring. Lowering the TES resistance also requires lowering the shunt resistance and increasing the bias current. Higher bias currents put tighter limits on the allowable resistance in the lowtemperature part of the wires leading to the bias circuit. None of these issues are insurmountable, but we need to consider their impact on the overall system design.

We presume, for the reasons discussed in the preceding paragraphs, that we will need to take as much of the available pixel area as possible for the TES itself, and that the TES optimization will deal with how to get the best performance out of that area. In time we will map the trade space between speed, size, and resolution. The other half of the challenge, then, is to place a thermal link and a wiring layer in the small remaining region around each TES, or to take those features out of the plane. Presently, we are investigating the feasibility of laying out everything except the absorbers in the plane.

Si-N membranes, typically 0.2 to $1 \mu \mathrm{m}$ thick, are now popular for use in low-temperature thermal isolation structures for bolometers, calorimeters, and other cryogenic devices. The $\mathrm{Si}-\mathrm{N}$ is typically used in $\mathrm{mm}$ scale continuous membranes and in beams with length $(0.1$ to $1 \mathrm{~mm})$ much greater than their widths. A survey of the literature on the low-temperature thermal conductivity of Si-N membranes shows that the geometrical scaling laws are not yet understood, even for the limited range of range of sizes presently being used. ${ }^{14,15,16}$ The confusion is caused by the competing effects of specular reflection and phonon mode exclusion. At low temperatures, the phonon mean free path in an insulating membrane exceeds the sample dimensions. Surface scattering dominates, and if the surface is smooth and free of defects the specular limit can be reached. (On the other hand, if the surface is rough, thermal transport remains diffusive.) In the specular limit, energy transport scales like radiative transfer. Only the cross-sectional area, and not the length, determines the thermal load. If a dimension becomes comparable to the phonon wavelengths, then the structure cannot support certain phonon modes, which are then unavailable for energy transport. Specular transport leads to higher thermal conductivities than expected from a bulk diffusive model, and mode exclusion leads to lower conductivities. These effects may contribute to our difficulty in determining the temperature dependence of the thermal conductance of our large Si-N membranes.

Though the existing measurements do not present a perfectly clear picture, they do support a specular model for the large area membranes and wide beams. ${ }^{17}$ This is encouraging, because it suggests that the weak link may not need to occupy much space. Unfortunately, none of these measurements were made on length scales even close to the $\sim 5 \mu \mathrm{m}$ spacing we'd like to use. For area $A$ and length $l$, in a competition between an effective conductance that scales as $A$ (specular) and one that scales as $A / l$ (diffusive), the $A / l$ term will eventually win as the length goes to zero. We want to know at what point that happens. We have designed TES test cells with a variety of compact designs for the weak link so that we can understand the relevant scaling. In some, the TES is surrounded by a perforated perimeter, making it look like a postage stamp. In others, a more "traditional" long beam suspension is used. Variations on those basic designs are represented. Newly fabricated devices will be tested soon.

\section{SUMMARY}

We have obtained energy resolution of $2.8 \mathrm{eV}$ at $1.5 \mathrm{keV}$ and $3.7 \mathrm{eV}$ at $3.3 \mathrm{keV}$ with a large, isolated TES pixel using a $\mathrm{Mo} / \mathrm{Au}$ proximity-effect bilayer on a silicon nitride membrane. An ideal calorimeter of the sensitivity and heat capacity of this device would have obtained $\sim 1.6 \mathrm{eV}$ resolution. Excess noise prevents reaching that theoretical benchmark. Nonetheless, this is a strong step forward for Mo/Au as a TES thermometer.

For use behind an x-ray telescope, such devices need to be arrayed with a pixel size and focal-plane coverage commensurate with the telescope focal length and spatial resolution. Since this requires fitting the TES and its thermal link into a far more 
compact geometry than has previously been investigated, we have outlined a set of experiments for understanding the basic scaling laws in TES pixel optimization.

\section{ACKNOWLEDGEMENTS}

We thank Kent Irwin and his colleagues at NIST-Boulder for the generous loan of several of their series-array SQUIDs. We are grateful to Barbara Mattson for her user-friendly data-acquisition software and to Carl Stahle for his work to coordinate

the fabrication efforts. We thank Rich Kelley, Andy Szymkowiak, and Harvey Moseley for comments and stimulating discussions on these results.

\section{REFERENCES}

1. C. K. Stahle, F. M. Finkbeiner, K. R, Boyce, T. Chen, E. Figueroa Feliciano, J. D. Gygax, R. L. Kelley, M. Li, B. J. Mattson, D. B. Mott, F. S. Porter, C. M. Stahle, A. E. Szymkowiak, and N. Tralshawala, "First Results from Mo/Au Transition-Edge Sensor X-ray Calorimeters," NIM A, 444, pp. 224-227, 2000.

2. C. J. Smithells, Metals Reference Book, Butterworths, Washington, 1962.

3. M. Hansen, Constitution of Binary Alloys, McGraw-Hill NY, 1958.

4. N. Tralshawala, S. Aslam, R. P. Brekosky, T. C. Chen, E. Figueroa Feliciano, F. M. Finkbeiner, M. J. Li, D. B. Mott, C. K. Stahle, and C. M. Stahle,." Design and Fabrication of Superconducting Transition Edge X-ray Calorimeters", NIM A, 444, pp. 188-191, 2000.

5. D. W. Fischer and W. L. Baun, "Diagram and Nondiagram lines in K Spectra of Aluminum and Oxygen from Metallic and Anodized Aluminum", J. Appl. Phys., 36, pp. 534-537, 1965.

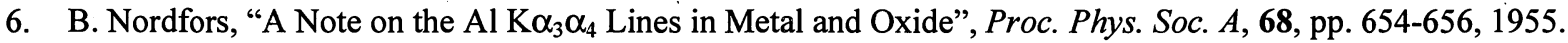

7. M. O. Krause and J. H. Oliver, "Natural Line Widths of Atomic K and L Levels, K $\alpha$ X-ray Lines and Several KLL Auger Transitions", J. Phys. Chem. Ref. Data, 8, pp. 329-338, 1979.

8. P. L. Lee and S. I. Salem, "Widths and Asymmetries of the $K \alpha_{1}$ and $K \alpha_{2} X$-ray Lines and the $\mathrm{L}_{2}$ and $\mathrm{L}_{3}$ Fluorescence Yields of the Transition Elements", Phys. Rev. A, 10, pp. 2027-2032, 1974.

9. K. D. Irwin, "An Application of Electrothermal Feedback for High-resolution Cryogenic Particle Detection", Appl. Phys. Lett., 66, pp. 1998-2000, 1995.

10. S.H. Moseley, J.C. Mather and D. McCammon, "Thermal Detectors as X-ray Spectrometers", J Appl. Phys. 56, pp. 1257-1262, 1984.

11. C. K. Stahle, D. McCammon, and K. D. Irwin, "Quantum Calorimetry", Physics Today, 52, pp. 32-37, 1999.

12. C. K. Stahle, et al., "Toward a 2-eV microcalorimeter x-ray spectrometer for Constellation-X", Proc. SPIE, 3765, pp. 82-93, 1999.

13. K. D. Irwin, G. C. Hilton, D. A. Wollman, and J. M. Martinis, "Thermal-response Time of Superconducting TransitionEdge Microcalorimeters", J. Appl. Phys., 83, pp. 3978-3985, 1998.

14. W. Holmes, J. M. Gildemeister, and P. L. Richards, "Measurements of Thermal Transport in Low Stress Silicon Nitride Films", Appl. Phys. Lett., 72, pp. 2250-2252, 1998.

15. M. M. Leivo and J. P. Pekola, "Thermal Characteristics of Silicon Nitride Membranes at Sub-Kelvin Temperatures", Appl. Phys. Lett., 72, pp. 1305-1307, 1998.

16. P. D. Mauskopf, J. J. Bock, H. Del Castillo, W. L. Holzapfel, and A. E. Lange, "Composite Infrared Bolometers with $\mathrm{Si}_{3} \mathrm{~N}_{4}$ Micromesh Absorbers", Appl. Opt., 36, pp. 765-771, 1997.

17. See Reference 14 . Also private communication, K. D. Irwin 